\title{
Anticancer Immunity and Cancer Immunotherapy with Cell Wall Skeleton and Related Synthetic Compounds
}

Recent studies on cancer immunology have been revealed that immunologial mechanism is playing roles in prevention of carcinogenesis and in suppression or regression of growth of cancer. The immunological mechanism of cell-mediated cytotoxicity to cancer cells has been elucidated on cellular and humoral bases. Killer T lymphocyte, activated macrophage and natural killer cells are considered to have cytotoxic activities against cancer cells.

\section{Cancer Immunotherapy and Its Evaluation}

On the immunological basis, many attempts have been made to find out immunotherapeutic modalities for cancer and there have been reported various kinds of trials of active, passive and non-specific immunotherapy of cancer. However, the principal guideline for the evaluation method of cancer immunotherapeutic agents has not yet been established. In 1979, the guideline for the evaluation of cancer immunotherapeutic agents was discussed in detail by two study groups, which were supported by the Cancer Research Grants from the Ministry of Health and Welfare, and Cooperative Cancer Research Program from the Ministry of Education, Science and Culture in Japan. The casic concepts for the evaluation method of cancer immunotherapeutic agents of both study groups are as follows.

1) Quality control of cancer immunotherapeutic agents should be made by the chemical and physicochemical and/or biological methods.

2) Antitumor activity of immunotherapeutic agents shoud be proved by using syngeneic transplantable tumor systems in mice and rats or guinea pigs.

3) Antitumor activity of cancer immunotherapeutic agents should be expressed via immunological bases in tumor-bearing hosts but not via direct cytotoxicity to tumor cells.

4) For the clinical evaluation of cancer immunotherapeutic agents, statistically welldesigned randomized trial is essential. Final criteria for the clinical evaluation of cancer immunotherapeutic agents should be made by the prolongation of survival periods of patients.

\section{Anticancer Immunity and BCG Cell Wall Skeleton}

It last decades, living cells of BCG have been extensively used as one of the most effective immunopotentiatiors in the immunotherapy of human cancers and experimental tumor systems, although various serious complications due to treatment with living BCG were reported.

We have shown that cell-wall skeletons (CWS) isolated from mycobacteria, nocardia, and corynebacteria have potent immunopotentiating activities inducing cell-mediated cytotxicities against syngeneic and autochtonous tumor systems in mice, rats and guinea pigs.

BCG-GWS is composed from three major components, i. e. mycolic acid, arabinogalactan and mucopeptide.

Anti-tumor activity of BCG-CWS on tranplantable tumors in syngeneic hosts and autcchthonous tumor system was proved in such experimental systems as mastocytoma P815-X2, EL-4, MH-134, melanoma $\mathrm{B} 16$ of mice and line 10 of straine 2 guinea pig. Both suppression and regression of experimental tumors were observed and systemic immunity to tumor was demonstrated in host in which tumor growth was suppressed.

The prophylactic effects of BDG-GWS on chemical carcinogensis of lung cancer were 
clearly demonstrated in rabbits and mice. BCG-GWS has been applied for the immunotherapy of human cancers such as lung cancer, leukemia, malignant melanoma and other neoplastic diseases in Japan. Prolongation of survival period of the patients was proved statistically in lung cancer, stage III of squamous cell carcinoma and adenocarcinoma, small cell carcinoma, large cell carcinoma and malignat pleurisy by the comprative studies on BCG-CWS treated group and historical control group.

Complications and side-effects in BCG-CWS treated patients were not serious and administration of BCG-CWS was well tolerated.

\title{
Antitumor Immunity and Nocardia Cell Wall Skeleton
}

Among the cell wall skeletons of various kinds of microorganisms related to mycobacteria, such as nocardia and corynebacteria, the cell wall skeleton of Nocardia rubra has been shown to be most effective in the immunotherapy of cancer in experimental syngeic transplantable tumors and autochthonous systems. The princ!pal chemical structure of $\mathrm{N}$. rubra cell wall skeleton is composed of nocardomycolic acid, arabinogalactan and mucopeptide which are almost similar to that of BCG-CWS except for the molecular weight and content of nocardomycolic acid. The strain of N. rubra is non-pathogenic and easily cultivated in synthetic and non-synthetic media in a few days. Cell-wall skeleton of $\mathrm{N}$. rubra is less toxic than BCG-GWS.

Cell wall skeleton of $\mathrm{N}$. rubra was applied to the immunotherapy of the patients with lung cancer, malignant melanoma and leukemia. Rondomized clinical trials conducted by Yamada et al. has shown that $\mathrm{N}$. rubra cell wall skeleton was proved to be effective statistically in the prolongation of survival by combined treatment with chemotherapeutics on acute myelogenous leukemia patients.

\section{Synthetic N-Acetylmuramyldipeptide Derivatives}

It has been shown that the minimal adjuvantt-active subunit of cell wall of BCG is $\mathrm{N}$-acetyl-muramyl-L-alanyl-D-isoglutamine, which was confirmed by the study using synthetic $\mathrm{N}$-acetylmuramyldipede (MDP) and related compounds.

These synthetic MDP and related compounds, however, have no antitumor activity in any experimental tumor system, while they have potent immunopotentiating activities in humoral antibody formation and induction of delayed thype hypersensitivity.

More recently, the authors have synthesized the hydrophobic derivatives of $\mathrm{N}$-acetylmuramyldipeptids by the introduction of high molecular fatty acids such as mycolic acids into 6-O-position of muramic acid of MDP. The synthetic 6-O-mycoloyl-N-acetylmuramyldipeptide showed suppressive activity of tumor growth of syngeneic mice and guinea pigs. We could succeed in the synthesis of 2,3-dimethoxy-5-methyl-6-(9'-carboxynonyl)-1, 4-benzoquinone derivative of $\mathrm{N}$-acetylmuramyl-L-valyl-D-isoglutamine methyl ester named as "quinonyl-MDP-66", having potent adjuvant and antitumor activities in experimental tumor systems.

There have been reported number of cancer immunotherapy, experimentally and clinically. However, well disigned experimental and statistical evaluation should be made to reach a conclusion that any immunotherapy is effective as a modality for cancer treatment.

\author{
Yuichi Yamamura, M D \\ President of the Japanese Society of \\ Internal Medicine (1980) and \\ President of Osaka University
}

